\title{
ESTUDOS SOBRE OS SCIONINI NEOTROPICAIS DO GENERO FIDENA - O SUBGENERO LEPTOFIDENA - (DIPTERA, TABANIDAE, PANGONIINAE)
}

\section{PAULO IIDE*}

\author{
Universidade Federal Fluminense, Centro de Estudos Gerais, 24250 Niterói, RJ, Brasil
}

Studies on the Neotropical Scionini of the genus Fidena - The subgenus Leptofidena - (Diptera, Tabanidae, Pangoniinae) - The author redescribes the males of Fidena (Leptofidena) morio (Wulp, 1881) based on details of external morphology and of the dissected genitalia hitherto not studied.

$$
\begin{gathered}
\text { Key words: Diptera - Tabanidae - Pangoniinae - Scionini - Neotropical - Fidena - Leptofidena - } \\
\text { morphology - male genitalia }
\end{gathered}
$$

Entre os Scionini neotropicais que precisam ser revistos, destaca-se Fidena morio (Wulp, 1881), considerada por Fairchild (1967) a espécie-tipo do subgênero Leptofidena Kröber, 1930. A validade deste subgênero monotípico foi aceita provisoriamente por Bernardi (1977) ao descrever pela primeira vez o macho desta espécie e fêmeas com variações morfológicas. Este autor não dissecou exemplares nem complementou o texto com ilustrações, julgando suficientes as figuras de Kröber (1930) e Fairchild (1967), embora não representem aspectos morfológicos de exemplares machos.

Ao reformular a sistemática dos Tabanidae, Mackerras (1955) considerou ser essencial a realizaçāo de dissecaçōes. Seus trabalhos, bem ilustrados, evidenciaram a sua preocupação em esclarecer os diversos aspectos morfológicos, evitando dúvidas que um texto meramente descritivo poderia suscitar. No trabalho de 1955 , incluiu Leptofidena entre os quatro gêneros de Pangoniinae que não conseguiu situar nas tribos do seu sistema de classificação, por não ter exemplares para examinar e dissecar, apoiandose apenas nas descrições originais.

Pelos motivos expostos, consideramos importante a redescrição dos machos desta espé-

\footnotetext{
Trabalho realizado sob os auspícios do Conselho $\mathrm{Na}$ cional de Desenvolvimento Científico e Tecnológico (CNPq) e com os recursos remanescentes do Instituto Biomédico da UFF.

*Correspondencia: Estrada do Pau-Ferro, 1042, 22743 Rio de Janeiro, RJ, Brasil.
}

cie, seguindo os critérios de Mackerras e que adotamos em nossos trabalhos sobre espécies brasileiras do gênero Fidena.

\section{MATERIAL E MÉTODOS}

O homótipo macho desta espécie nos foi gentilmente cedido para estudo pelo Dr. Graham Bell Fairchild (Florida Department of Agriculture \& Consumer Services - USA) e por ele comparado em 1964 com o exemplar que considerou como tipo de Pangonia morio, depositado no Zoologische Museum der Universiteit van Amsterdam (Holanda), e em 1965 com o tipo de Leptofidena beelzebul que foi remetido pelo Zoologisches Institut, Martin Luther Universität, Halle Saale (Alemanha Oriental). $O$ outro exemplar, pertencente à coleção de Diptera do Museu de Zoologia da Universidade de São Paulo (Brasil), cedido por cortesia do Dr. Nelson Papavero, é sem dúvida o mesmo descrito por Bernardi em 1977 embora este autor não tenha citado o coletor do material, talvez por estar quase ilegível no rótulo mas que consideramos como sendo "Neghme col.". Outros dados sobre equipamento, material e metodologia constam em nossos trabalhos anteriores sobre o gênero Fidena (Iide, 1982a, b; 1988).

\section{REDESCRIÇÃO}

\section{Gênero Fidena Walker Subgênero Leptofidena Kröber}

Leptofidena Kröber, 1930: 211 (chave), 222 (descrição como gênero), : 223 (descriçāo 
da espécie-tipo L. beelzebul; possivel inclusão de Erephopsis submetallica Brèthes neste gênero). Mackerras, 1955: 457, 458 (gênero nāo situado nas tribos de Pangoniinae; caracteres).

Fidena (Leptofidena), Fairchild, 1967 (1966): 376 (como subgênero de Fidena; L. beelzebul na sinonímia de Pangonia morio Wulp, 1881); 1969: 204 (como subgênero monotípico; caracteres),: 227 (chave); 1971: 25 (catálogo). Bernardi, 1977: 26i (validade do subgênero; discussão).

Fidena (Leptofidena) morio (Wulp, 1881) (Figs. 1 a 17)

Pangonia morio Wulp, 1881: 156 (holótipo fêmea; Argentina).

Diatemineura morio, Ricardo, 1900: 172 (catálogo). Kertész, 1908: 170 (catálogo).

Erephopsis morio, Brèthes, 1910: 473 (caracteres).

Leptofidena beelzebul Kröber, 1930: 223 (holótipo fêmea; Argentina - Tucuman), Fig. 6 (palpos, antena e fronte); 1930a: 134 (Norte da Argentina); 1934: 245 (catálogo). Barreto \& Duret, 1954: 203 (redescrição; Argentina Província de Buenos Aires: Zona da Bahia Blanca, Estância Barrau; diferenciação de $E$. submetallica). Fairchild, 1967 (1966): 352 (caracteres do holótipo; na sinonímia de P. morio), Fig. 14 (asa, cabeça, fronte, antena e palpo). Coscaron, 1967: 105 (lista; nota de sinonímia).

Fidena morio, Kröber, 1933: 242 (chave): 264 (redescrição: Argentina - Mendoza), Fig. 13 (fronte, palpo e antena). (NOTA: Possivelmente nāo é $P$. morio); 1934: 245 (catálogo). Coscaron, 1967: 108 (lista; L. beelzebul na sinonímia com base em Fairchild, 1967).

Fidena (Leptofidena) morio, Fairchild, 1967 (1966): 352 (comparação com L. beelzebul): 376 (beelzebul na sinonímia); 1967a: 249 (caracteres do holótipo; identificação de um exemplar macho); 1969: 204 (caracteres); 1971: 25 (caracteres); 1971: 25 (catálogo). Bernardi, 1977: 263 (redescrição de fêmeas com variações): 265 (descrição do macho), (Argentina - Buenos Aires: Estância Barrau; Mendoza: Uspallata. Chile - Valdívia: Panguipulli).

\section{HOMÓTIPO - Macho - Comprimento total $14 \mathrm{~mm}$.}

Aspecto geral: exemplar robusto, castanhoescuro a preto; cabeça e tórax foscos; abdômen brilhante. Pilosidade castanha a preta, mais densa na cabeça e principalmente no tórax; esparsa no abdômen. Palpos cilindroides. Asas quase hialinas, levemente acinzentadas; célula $R_{5}$ fechada com a veia $R_{5}+M_{1}$ um pouco mais longa que $1 / 4$ do comprimento da $M_{1}$ e o dobro da transversa $\mathrm{r}-\mathrm{m}$. Genitália semelhante a de outras espécies do gênero Fidena.

Cabeça castanho-escura e fosca, com densa polinosidade castanha a ferrugínea, principalmente no triângulo frontal onde há um tufo de pêlos pretos. Face com longos pêlos castanhos, progressivamente maiores em direção às genas, onde são mais longos, mais densos e mais escuros ("barba"). Projeção facial em forma de cone truncado (Fig. 1); em vista de perfil o comprimento da face (menor distância da margem ocular até a base da probóscida) corresponde à cerca de 0,81 da sua altura (distância da base dos palpos até a base das antenas). Triângulo ocelar preto com ocelos amarelados; pêlos pós-ocelares longos e pretos. Pilosidade ocular densa e castanha com reflexos cúpreos; em vista de perfil a largura dos olhos corresponde à cerca de $0,6 \mathrm{da}$ altura; margem ocular (órbitas) com uma faixa de polinosidade um pouco mais clara que a da face, tornando-se acinzentada-clara com leves reflexos prateados em parte das genas e no occipício.

Antenas castanho-escuras; escapo e pedicelo quase pretos com polinosidade castanha; flagelo castanho revestido de polinosidade ferrugínea com reflexos mais claros. O escapo tem o dobro do comprimento do pedicelo e é um pouco mais robusto (Fig. 2). O 8 \% flagelômero é um pouco mais longo que a soma do comprimento do 6 ? e 7 ? , porém um pouco mais curto que o 1 . (Figs. 2 e 3 ). $\mathrm{Na}$ extremidade do flagelo há um conjunto de pêlos pretos e longos com cerca de $4 / 5$ do comprimento do 8 ? flagelômero (Fig. 3).

Probóscida ligeiramente mais longa que a metade do comprimento do exemplar $(0,52 \mathrm{x})$ e quase igual ao dobro da altura da cabeça $(1,95$ $x)$. Palpos castanho-escuros e brilhantes, com densos pêlos pretos; os dois segmentos são aproximadamente cilindróides, com o $1^{\circ}$. tendo cerca de $2 / 3$ do comprimento do 2 ? ; no $2^{\circ}$. segmento 


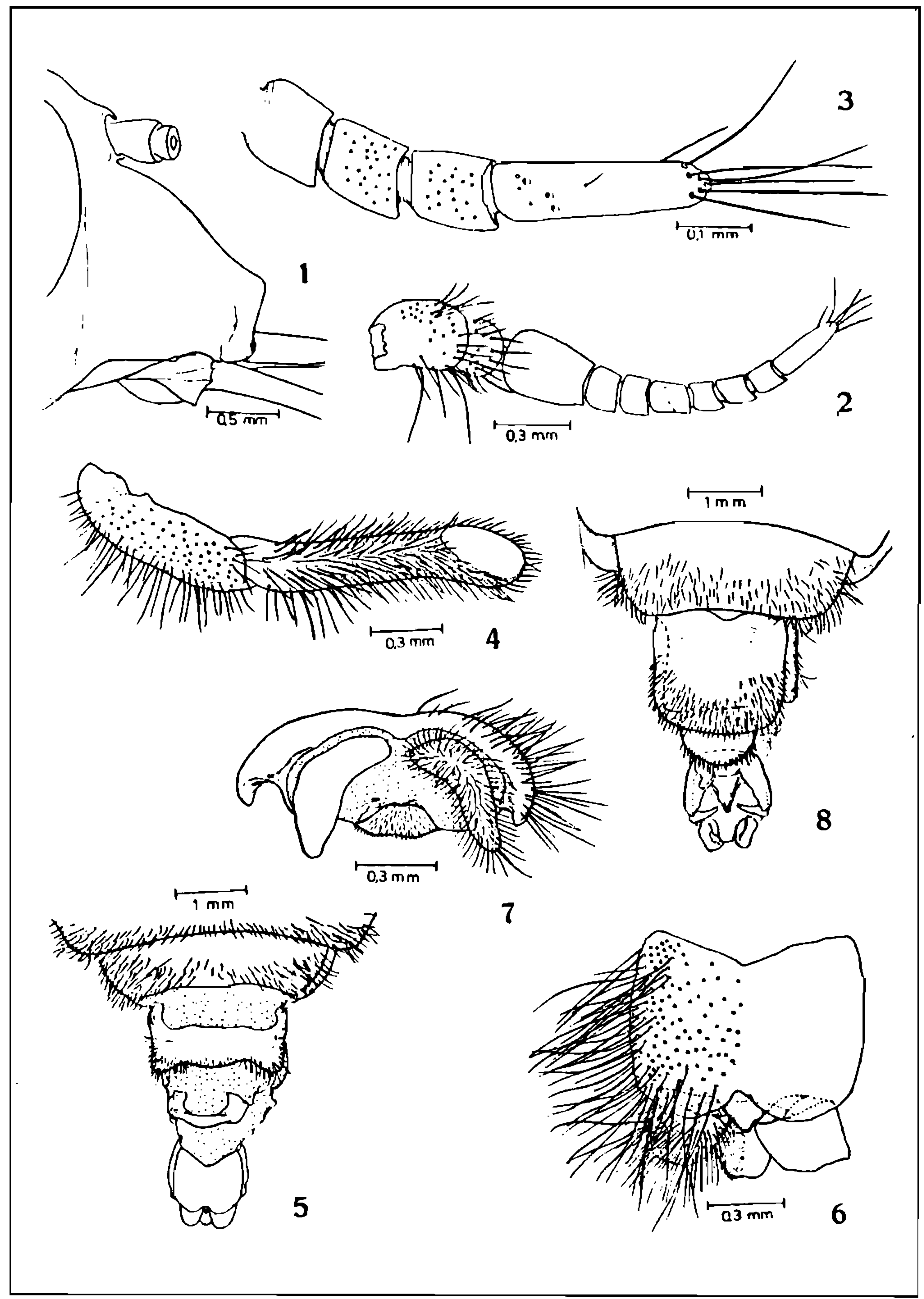

Fidena (Leptofidena) morio (Wulp, 1881), macho - Fig. 1: face, vista lateral. Fig. 2: antena, vista medial. Iig. 3: idem, detalhe dos últimos flagelômeros. Fig. $4:$ palpo, vista lateral. Fig. $5:$ últimos segmentos abdominais, vista dorsal. Fig. 6:90 tergito (epandrium), cerci e 109 esternito, vista dorsal. Fig. 7 : idem, vista posterior. Fig. 8 : últimos segmentos abdominais, vista ventral. 
- 1/4 apical é ligeiramente dilatado (Fig. 4), com uma área deprimida de contorno elipsóide e de cor mais clara.

Tórax castanho-escuro, quase totalmente fosco, com densa polinosidade castanha, um pouco mais escura ao longo das linhas dorsocentrais e mediana, e nas partes laterais da área pré-sutural. $O$ escudo é revestido de pêlos castanho-escuros a pretos, mais longos e mais densos nos limites da área pré-sutural com a mesopleura (anepisterno), na margem posterior do calo pós-alar e antes da sutura escuto-escutelar. Os calos umerais (pós-pronoto) e pós-alares e também a margem posterior do escutelo, são de tonalidade castanha mais clara. Pêlos pleurais longos, castanho-escuros a pretos, mais densos na metade dorsal da mesopleura (anepisterno) e da pteropleura (anepímero), na metapleura (catetergito) e na parte anterior da esternopleura (catepisterno).

Asas semi-hialinas, tenuemente acinzentadas, com tonalidades castanhas na base, nas células costal e sub-costal, e ao longo dos lados da veia $R_{1}$. Célula $R_{5}$ fechada com a veia $R_{5}+M_{1}$ cerca de duas vezes mais longa que o comprimento da transversa $\mathrm{r}-\mathrm{m}$ ou da $\mathrm{CuA} \mathrm{A}_{2} \mathrm{~A}$, e ainda com 0,27 do tamanho da $M_{1}$. A base da veia $R_{4}$ é angulosa e o seu apêndice é curto, com $2 / 3$ do comprimento da $\mathrm{r}-\mathrm{m}$. Halteres castanhos com capitelo castanho-claro a alaranjado.

Pernas castanho-escuras com pêlos pretos e longos nas coxas, mais abundantes no 1 ? par. Nos fêmures os pêlos têm distribuição mais uniforme e são progressivamente mais curtos em direçāo à extremidade distal. Tíbias e tarsos revestidos de pêlos curtos e castanhos, com reflexos castanho-claros. Articulação fêmur-tibial castanho-clara a amarelada. Tíbias e tarsos revestidos de pêlos curtos e castanhos, com reflexos castanho-claros. Empódio e pulvilos amarelo-claros, ladeados por garras pretas. $\mathrm{O}$ revestimento polinoso das pernas varia do castanho ao ferrugíneo.

Abdômen robusto com a largura correspondendo a cerca de 0,8 do comprimento. Coloração castanho-escura a quase preta e brilhante; pêlos pretos poucos numerosos, mais abundantes nas margens laterais e nos ângulos láteroposteriores dos tergos. Polinosidade discreta, mais densa ventralmente. No pós-abdômen o 7 ? tergito tem a margem posterior ligeiramente sinuosa com lobos laterais salientes e com pêlos mais desenvolvidos (Fig. 5); o $8^{\circ}$ tergito é arqueado, com expansões laterais angulosas. $\mathrm{O}$ 79 esternito é sub-retangular, ligeiramente mais longo do que largo; a sua margem posterior é em curvatura contínua com as margens laterais; o revestimento piloso estende-se dos $2 / 5$ posteriores até mais da metade das áreas laterais (Fig. 8); o 8? esternito é aproximadamente semi-circular, piloso só na metade posterior.

Genitälia - O 99 tergito (epandrium) é subretangular, mais largo do que longo e com margens laterais moderadamente encurvadas (Figs. 5 e 6); a margem anterior é em forma de um " $V$ " com os ramos formando um ângulo obtuso de aproximadamente $140^{\circ}$; a margem posterior tem uma profunda reentrância mediana (Figs. 6 e 9). Cerci curtos, largos e fortemente encurvados, com revestimento piloso mais curto que o do $9^{\circ}$. tergito. $09^{\circ}$ esternito tem as margens laterais (nos gonocoxitos) ligeiramente encurvadas, em continuidade com a curvatura mais acentuada da margem anterior (Fig. 11). Na faixa basal do 9 ? esternito há uma placa mediana ventral (hypandrium) glabra e de contorno aproximadamente semicircular (Fig. 17). Forcipes inferiores (gonostyli) simples e alongados, mais estreitos no quarto distal que é digitiforme, um pouco torcido e ligeiramente encurvado (Figs. 11 e 12); e margem posterior é levemente sinuosa com uma angulosidade perto da base; 0 revestimento piloso é muito mais curto do que o da face interna dos gonocoxitos (Figs. 11 e 13). Aedeagus (Fig. 14) de aspecto geral semelhante ao observado em outras espécies de Fidena; a extremidade distal dos flagelos é ligeiramente dilatada e encurvada (Figs. 15 e 16), exteriorizando-se da bainha do pênis por uma abertura posterior e uma fenda ventral (Fig. 16). $010^{\circ}$. esternito é subtrapezoidal com os lados formando entre si um ângulo de aproximadamente $45^{\circ}$; a sua margem posterior tem uma ligeira depressão mediana e situa-se praticamente ao nível da margem posterior dos cerci (Figs. 9 e $10)$.

Caracteres não mencionados na descrição original de Bernardi (1977) - No exemplar procedente do Museu de Zoologia da Universidade de São Paulo, a ausência dos flagelos antenais e da maior parte dos segmentos das pernas, além da mutilação parcial dos palpos e da probóscida, impossibilitaram uma descrição mais completa por Bernardi e a comparação destas estruturas com as do homótipo. A fragmentação do 


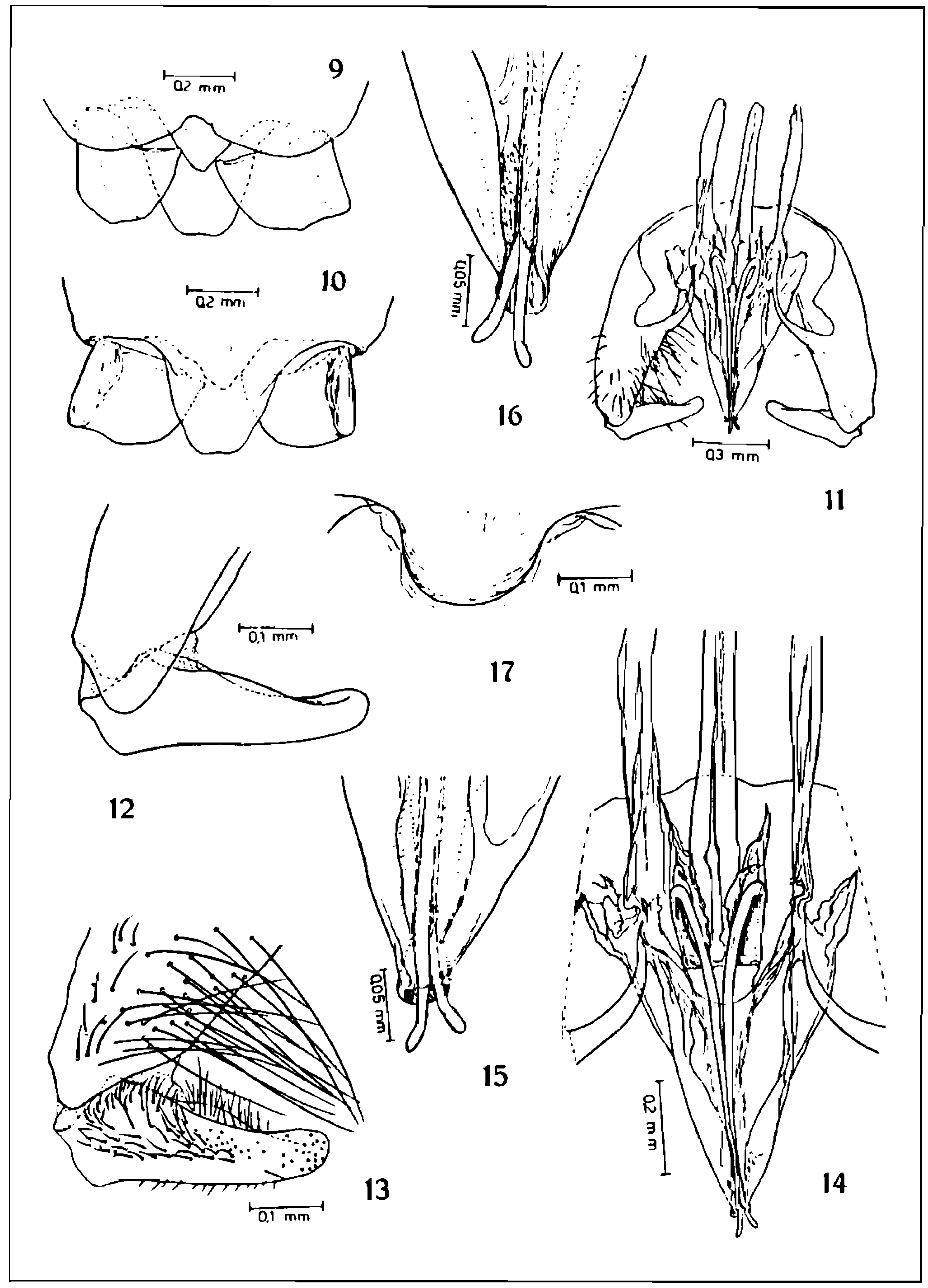

Filena (Leptofidena) morio (Wulp, 1881), macho - Fig. 9:109 esternito e cerci, vista dorsal. Fig. 10: idem, vista ventral. Fig. 11:99 esternito (hypandrium, aedeagus e gonopódios), vista dorsal. Fig. 12: forceps inferior (gonostylus), vista dorsal. Fig. 13: idem, vista ventral. Fig. 14 : aedeagus, vista dorsal. Fig. 15 : bainha do pênis e flagelos, detalhe da extremidade distal, vista dorsal. Fig. 16: idem, vista ventral. Fig. 17: placa mediana ventral do 9 o esternito (hypandrium), vista ventral. 
mesonoto no ponto de penetração do alfinete evidencia que o exemplar estava seco por ocasião da sua montagem, resultando nas alterações acima citadas e na colagem da cabeça muito perto do tórax e deslocada para a direita. Acreditamos que a pilosidade menos numerosa na parte superior dos olhos e na mesopleura (anepisterno) e ainda o menor comprimento do exemplar $(12 \mathrm{~mm})$, também são conseqüências da montagem acidentada.

Não obstante a pigmentação um pouco mais escura do corpo, o seu aspecto geral bem como o das asas é idêntico ao do homótipo. As pequenas variações que encontramos nas relaçōes métricas das veias alares, face, olhos e abdômen, são pouco significativas em espécies do gênero Fidena.

Exemplares examinados - Coleção Fairchild: Chile - 1 macho, Valparaiso, Reñaca, (Edwin P. Reed), 1918 (?); (Rótulos adicionais: "Fidena morio v. d. W., J. Beq. det.", "Homotype Pangonia morio Wulp", "Comp. w. I type of P. morio Wulp, Amsterdam, 1964, Good agreement" e "Comp. w. 9 type of Leptofidena beelzebul Kröb. in Mus. Halle 1965, Good agreement"). Coleção do Museu de Zoologia da Universidade de São Paulo: Chile - 1 macho, Panguipulli, Valdivia, (Neghme col.).

Distribuiçāo geográfica - Argentina - Tucuman; Mendoza (?); Buenos Aires (Bahia Blanca). Chile - Valparaiso (Reñaca); Valdivia (Panguipulli).

\section{COMFNTÄRIOS}

O estudo morfológico da genitália não evidenciou aspectos relevantes que caracterizem ou justifiquem a validade do subgénero Leptofidena. O 9'. tergito mais largo do que longo e o 9? esternito com as margens laterais e anterior em curvatura contínua, não parecem ser caracteres confiáveis enquanto permanecerem desconhecidas as estruturas da genitália na maior partc dos Scionini, e não sejam estabelecidas de modo amplo as diferenças e semelhanças entre os taxons das categorias inferiores desta tribo. A placa mediana ventral do 9 ? esternito, de forma típica para cada uma das espécies do subgênero Fidena que estudamos ou examinamos anteriormente (Iide, 1982a, b; 1988), não tem diferenças excepcionais que permitam dis. tinguir leptofidena como subgênero.
O 8? flagelômero muito longo e o 2 ? segmento dos palpos em forma de garra e sulcados lateralmente nos 2/3 basais (considerados como típicos para Leptofidena), são aspectos restritos apenas às fêmeas. $O$ comprimento da veia $\mathrm{R}_{5}+\mathrm{M}_{1}$ (relativamente longa, com cerca de 0,31 do comprimento da $M_{1}$ nas fêmeas e 0,27 nos machos) se não tiver variações como as que assinalamos em $F$. (F.) rufibasis Kröber, pode ser uma característica subgenérica auxiliar, desde que fundamentada no exame de um número maior de exemplares.

Pelos motivos expostos, apenas o contraste entre a pigmentação castanho-escura a preta do corpo e a presença de asas hialino-acinzentadas, bem como a célula $R_{5}$ fechada e pedunculada, e os palpos não achatados lateralmente, constituem o conjunto dos caracteres de consistência discutível que mantêm a validade do subgênero Leptofidena.

\section{AGR ADECIMENTOS}

Dedicamos o presente trabalho ao Prof. Dr. Hugo de Souza Lopes em reconhecimento ao zelo e atenção demonstrados ao orientar a nossa formação científica. Agradecemos também ao Dr. Graham Bell Fairchild e ao Dr. Nelson Papavero pelos exemplares cedidos para estudo.

\section{REFERENNCIAS}

BARRETTO, M. P. \& DURFT, J. P., 1954. Sobre alguns tabânidas argentinos, com a descriçāo de três novas espécies de Mycteromyia Phil. (Diptera, Tabanidae). Rev. Brasil. Ent., 1: 203-212.

BERNARDI, N., 1977. Notas sobre Tabanidae americanos (Diptcra). II. O subgênero Leptofidena Kröber. Papéis Avulsos Dep. Zool. S. Paulo, 30 : 216-265.

BRETHES, J., 1910. Dipteros nuevos ó poco conocidos de Sud-América. An. Mus. Nac. Buenos Aires, 20 (= Ser. 3, Tomo 13): 469-484.

COSCARÓN, S., 1967. Elenco sistemático de Tabanidac Argentina (Diptera, Insecta). Seg. Jorn. Entomoepid. Arg. I (1965), 1: 105-131.

l:AIRCHILD, G. B., 1967a. Notes on Neotropical Tabanidac. 1X. The species described by Otto Kröber. Studia Ent., Rio de Janeiro, 9: 329-384, (1966).

FAIRCHILD, G. B., 1967b. Notes on Neotropical Tabanidac (Diptera). X. The species described by J. R. Schiner and others. Pacific Insects, 9: 243256.

IA AIRCHILD, G. B., 1969. Notes on Neotropical Tabanidac. XII. Classification and distribution, with keys to genera and subgenera. Arq. Zool. S. Paulo, 17: 199-255.

FAIRCHILD, G. B., 1971. A catalogue of the Diptera of the Americas South of the United States. 28. 
ramily Tahanidae. Museu de Zoologia, Universidade de São Paulo. 168 p.

IIDL, P., 1982a. Estudo sobre a morfologia, a terminologia e o valor taxonômico das estruturas da genitália masculina dos Tabanidac, com base em Fidena (Fidena) sorbens (Wiedemann, 1828). (Diptera, Tabanidae, Pangoniinae). Rev. Brasil. Biol., 42: 197-211.

IIDL, P., 1982b. Fstudos morfológicos e taxonômicos sobre os Scionini brasileiros. O gênero Fidena. Parte I. (Diptera, Tabanidae, Pangoninac). Rev. Brasil. Biol., 42: 347.358.

IIDL:, P., 1988. Fstudos morfológicos e taxonômicos sobre os Scionini brasileiros. O gênero Fidena. Parte II. (Diptera, Tabanidae, Pangoniinae). Rer. Brasil. Biol., 48: 139-154.

KLETİSZ, C., 1908. Catalogus dipterorum hucusque descriptorum. 3. Lipsiate, Budapestini (= Leipzig. Budapest), $367 \mathrm{p}$.

KROBLR, O., 1930. Dic Tribus Pangoniini der neotro- pische Region. Zool. Anz., 89: 211-228.

KRÖBER, O., 1930a. Tabanidae, p. 106-161. In British Muscum (Natural History), Diptera of Patagonia and South Chile 5 (2), 197 p. London.

KRÖBF,R, O., 1933. Die neotropischen Arten der Tabanidengattung Fidena Walk. Arch. Naturgesch. (N. F.) 2: 231-284

KRÖBER. O., 1934. Catálogo dos Tabanidae da América do Sul e Central, incluindo o México e as Antilhas. Rev. Ent., Rio de Janeiro, 4: 222-276, 291 333.

MACKERRAS, I. M., 1955. The classification and distribution of Tabanidae (Diptera). II. History: Morphology: Classification:Subfamily Pangoniinae. Aust. J. Zool., 3:439-511.

RICARDO. G., 1900. Notes on the Pangoninae of the family Tabanidac in the British Museum Collection. Ann. Mag. Nat. Hist., 5: 97-121, 168-182.

WULP, F. M. VAN DER, 1881. Amerikaansche Diptera. Tijdschr. Ent., 24: 141-168. 